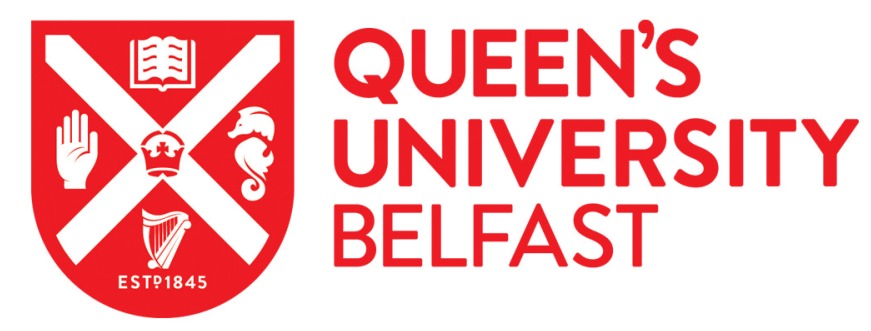

\title{
Hyperfine interaction in the ground state of the negatively charged nitrogen vacancy center in diamond
}

Felton, S., Edmonds, A. M., Newton, M. E., Martineau, P. M., Fisher, D., Twitchen, D. J., \& Baker, J. M. (2009). Hyperfine interaction in the ground state of the negatively charged nitrogen vacancy center in diamond. Physical Review B (Condensed Matter), 79(7), [075203]. https://doi.org/10.1103/PhysRevB.79.075203

Published in:

Physical Review B (Condensed Matter)

Document Version:

Publisher's PDF, also known as Version of record

Queen's University Belfast - Research Portal:

Link to publication record in Queen's University Belfast Research Portal

Publisher rights

(C) 2009 The American Physical Society

\section{General rights}

Copyright for the publications made accessible via the Queen's University Belfast Research Portal is retained by the author(s) and / or other copyright owners and it is a condition of accessing these publications that users recognise and abide by the legal requirements associated with these rights.

Take down policy

The Research Portal is Queen's institutional repository that provides access to Queen's research output. Every effort has been made to ensure that content in the Research Portal does not infringe any person's rights, or applicable UK laws. If you discover content in the Research Portal that you believe breaches copyright or violates any law, please contact openaccess@qub.ac.uk. 


\title{
Hyperfine interaction in the ground state of the negatively charged nitrogen vacancy center in diamond
}

\author{
S. Felton, A. M. Edmonds, and M. E. Newton* \\ Department of Physics, University of Warwick, Coventry, CV4 7AL, United Kingdom \\ P. M. Martineau and D. Fisher \\ DTC Research Centre, Belmont Road, Maidenhead, Berkshire, SL6 6JW, United Kingdom \\ D. J. Twitchen \\ Element Six Limited, King's Ride Park, Ascot, Berkshire, SL5 8BP, United Kingdom \\ J. M. Baker \\ Oxford Physics, Clarendon Laboratory, Oxford, OX1 3PU, United Kingdom
}

(Received 26 October 2008; revised manuscript received 1 January 2009; published 12 February 2009)

\begin{abstract}
The ${ }^{14} \mathrm{~N},{ }^{15} \mathrm{~N}$, and ${ }^{13} \mathrm{C}$ hyperfine interactions in the ground state of the negatively charged nitrogen vacancy $\left(\mathrm{NV}^{-}\right)$center have been investigated using electron-paramagnetic-resonance spectroscopy. The previously published parameters for the ${ }^{14} \mathrm{~N}$ hyperfine interaction do not produce a satisfactory fit to the experimental $\mathrm{NV}^{-}$ electron-paramagnetic-resonance data. The small anisotropic component of the $\mathrm{NV}^{-}$hyperfine interaction can be explained from dipolar interaction between the nitrogen nucleus and the unpaired-electron probability density localized on the three carbon atoms neighboring the vacancy. Optical spin polarization of the $\mathrm{NV}^{-}$ ground state was used to enhance the electron-paramagnetic-resonance sensitivity enabling detailed study of the hyperfine interaction with ${ }^{13} \mathrm{C}$ neighbors. The data confirmed the identification of three equivalent carbon nearest neighbors but indicated the next largest ${ }^{13} \mathrm{C}$ interaction is with six, rather than as previously assumed three, equivalent neighboring carbon atoms.
\end{abstract}

DOI: 10.1103/PhysRevB.79.075203

\section{INTRODUCTION}

The negatively charged nitrogen vacancy $\left(\mathrm{NV}^{-}\right)$color center in diamond has an $S=1$ ground state and a zerophonon line (ZPL) at $1.945 \mathrm{eV}(637 \mathrm{~nm})$. It was first observed using electron paramagnetic resonance (EPR) by Loubser and van $\mathrm{Wyk}^{1}$ in 1977. In recent years there has been renewed interest in this defect $(>150$ papers since 2000) mainly focused on developing quantum information technologies, with $\mathrm{NV}^{-}$suggested as a possible qubit in solid-state quantum computation ${ }^{2}$ and a single-photon source for quantum communication. ${ }^{3}$ A detailed understanding of the properties and different charge states of the NV centers is important for these applications; one step in this process is the detection of an EPR signal from the neutral nitrogen vacancy $\left(\mathrm{NV}^{0}\right)$ which was recently reported. ${ }^{4}$ Recent theoretical calculations of hyperfine parameters in the $\mathrm{NV}^{-}$center by Gali et $a .^{5}$ provide important insight into the electronic structure of the NV center.

The nitrogen vacancy center consists of a substitutional nitrogen atom with a nearest-neighbor vacant carbon site (vacancy) (see Fig. 1). The defect has trigonal $\left(C_{3 v}\right)$ symmetry around the crystallographic $\langle 111\rangle$ direction connecting the nitrogen and the vacancy. The defect-molecule approach, where the properties of the defect are assumed to be determined by the electrons in the dangling orbitals on the atoms neighboring the vacancy, ${ }^{6}$ can be used to model the NV center. The $C_{3 v}$ point group requires that the NV molecular orbitals transform as $a_{1}, a_{1}$, and $e$. One of the $a_{1}$ singlets is derived from the nitrogen dangling orbital $\left(a_{1 \mathrm{~N}}\right)$, the other from a symmetric combination of $\mathrm{C}_{a}$ dangling orbitals $\left(a_{1 \mathrm{C}}\right)$, and the $e$ doublet from antisymmetric combinations of the $\mathrm{C}_{a}$
PACS number(s): 81.05.Uw, 61.72.jn, 76.30.Mi

dangling orbitals. For $\mathrm{NV}^{-}$there are six electrons to accommodate in these orbitals. Assuming that the energies of these levels order as $a_{1 \mathrm{~N}}<a_{1 \mathrm{C}}<e$, the lowest energy configuration is $a_{1 \mathrm{~N}}^{2} a_{1 \mathrm{C}}^{2} e^{2}$ which gives rise to the many-electron states ${ }^{1} A_{1}$, ${ }^{3} A_{2}$, and ${ }^{1} E$. Promotion of one electron from a singlet to the doublet gives the configuration $a_{1 \mathrm{~N}}^{2} a_{1 \mathrm{C}}^{1} e^{3}$, and the manyelectron states ${ }^{1} E$ and ${ }^{3} E$. EPR and optical spectroscopy have shown the ZPL at $1.945 \mathrm{eV}$ to arise from a ${ }^{3} A_{2}$ to ${ }^{3} E$ transition, with ${ }^{3} A_{2}$ being the ground state. ${ }^{7,8}$ Determining the ordering of the energy levels is a challenging theoretical problem; at least one other state, which dramatically impacts on the photophysics of this defect, is located between the ${ }^{3} A_{2}$ and ${ }^{3} E$ states. ${ }^{8,9}$ Using spin-polarized local densityfunctional cluster theory, Goss et al. ${ }^{10}$ calculated the energy-

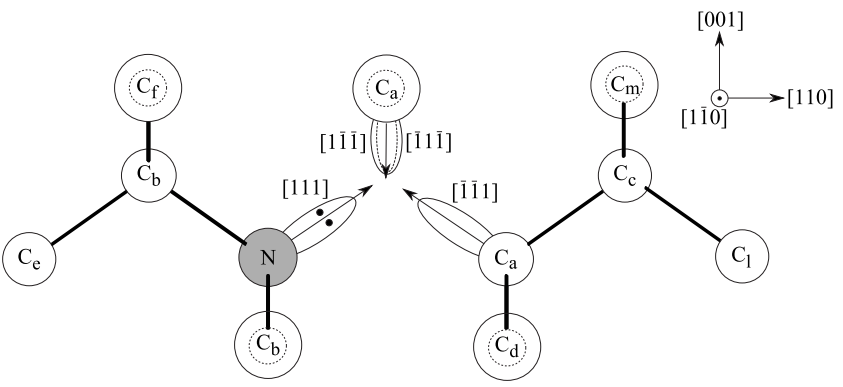

FIG. 1. Projection of the nitrogen vacancy center in diamond on the $(1 \overline{1} 0)$ plane. The grey disc represents a nitrogen atom and the other circles represent carbon atoms. The larger circles represent carbon atoms located above the plane of the paper and the smaller dashed circles represent carbon atoms below the plane of the paper. The labels on the carbon atoms denote sets of equivalent neighbors. 
TABLE I. Summary of sample preparation and quantitative EPR results for the samples investigated.

\begin{tabular}{lccc}
\hline \hline & Sample A & Sample B & Sample C \\
\hline Synthesis method & HPHT & HPHT & CVD \\
Nitrogen isotope distribution & $99.6 \%{ }^{14} \mathrm{~N}$ & $\sim 95 \%{ }^{15} \mathrm{~N}$ & $\sim 90 \%{ }^{15} \mathrm{~N}$ \\
As grown $\left[\mathrm{N}_{S}^{0}\right] / \mathrm{ppm}$ & $\sim 10$ & $\sim 150$ & $\sim 2$ \\
HPHT annealed & $\mathrm{No}$ & Yes & $\mathrm{No}$ \\
Post HPHT annealing $\left[\mathrm{N}_{S}^{0}\right] / \mathrm{ppm}$ & & $24(2)$ & \\
Irradiation type & $1.9 \mathrm{MeV} e^{-}$ & $1.5 \mathrm{MeV} e^{-}$ & $1.9 \mathrm{MeV} e^{-}$ \\
Irradiation dose & $3 \times 10^{18} \mathrm{~cm}^{-2}$ & $4 \times 10^{17} \mathrm{~cm}^{-2}$ & $6 \times 10^{17} \mathrm{~cm}^{-2}$ \\
Post irradiation annealing & $4 \mathrm{~h}$ at $1100 \mathrm{~K}$ & $4 \mathrm{~h}$ at $1100 \mathrm{~K}$ & $>4 \mathrm{~h} \mathrm{at} 1100 \mathrm{~K}$ \\
Post irradiation and $1100 \mathrm{~K}$ annealing $\left[\mathrm{N}_{S}^{0}\right] / \mathrm{ppm}$ & $1.2(1)$ & $17(1)$ & $0.8(1)$ \\
Post irradiation and $1100 \mathrm{~K}$ annealing $\left[\mathrm{NV}^{-}\right] / \mathrm{ppm}$ & $1.0(1)$ & $0.6(1)$ & $0.12(1)$ \\
\hline \hline
\end{tabular}

level ordering as ${ }^{3} A_{2},{ }^{1} E,{ }^{1} A_{1}$, and ${ }^{3} E$ with an optical energy of $1.77 \mathrm{eV}$ for the ${ }^{3} A_{2} \rightarrow{ }^{3} E$ transition. In contrast, in a recent study by Gali et al.,${ }^{5}$ using a different variation in densityfunctional theory (DFT) calculations, the energy levels are found to order as ${ }^{3} A_{2},{ }^{1} A_{1},{ }^{1} E$, and ${ }^{3} E$ with a value of 1.71 $\mathrm{eV}$ for the optical transition. This is also the energy-level scheme suggested by Manson and McMurtrie ${ }^{11}$ to be compatible with the observed spin-polarization behavior of the $\mathrm{NV}^{-}$center, where a nonspin conserving intersystem crossing between the ${ }^{3} E$ and ${ }^{1} A_{1}$ states leads to the preferential population of the $M_{S}=0$ level of the ${ }^{3} A_{2}$ state, given the experimentally determined positive sign of the zero-field splitting $D .{ }^{12}$ This ordering of the energy levels is confirmed by Rogers et al.: ${ }^{13}$ they have associated an infrared ZPL at $1046 \mathrm{~nm}$ with a transition within the $\mathrm{NV}^{-}$center and concluded from uniaxial stress measurement on this ZPL that it is due to an ${ }^{1} A_{1} \rightarrow{ }^{1} E$ transition.

In this paper we re-examine the negatively charged nitrogen vacancy and present experimental results from EPR measurements on $\mathrm{NV}$ centers in both ${ }^{14} \mathrm{~N}$-doped and ${ }^{15} \mathrm{~N}$-doped diamond. The isotopical substitution of ${ }^{14} \mathrm{~N}$ with ${ }^{15} \mathrm{~N}$ simplifies the EPR spectrum allowing the ${ }^{13} \mathrm{C}$ hyperfine interactions to be more easily studied. These interactions of the electron spin with surrounding nuclei are of particular interest since it has been suggested that they could be used to extend the number of qubits per node for quantum computation with NV centers. ${ }^{14}$ This investigation is motivated by the recent theoretical predictions of Gali et al., ${ }^{5}$ which provide insight into the electronic structure of $\mathrm{NV}^{-}$, and challenge the voracity and interpretation of the hyperfine spinHamiltonian parameters determined in previous experimental studies. An improved understanding of $\mathrm{NV}^{-}$is essential both for full exploitation of this remarkable color center and to guide the search for systems with even more attractive properties, e.g., emission in infrared, neutral centers, etc.

\section{EXPERIMENTAL}

The NV center in diamond can be produced in a number of different ways. For example, NV centers are formed in diamonds containing single substitutional nitrogen $\left(\mathrm{N}_{S}\right)$ by irradiation damage (creating vacancies) and subsequent an- nealing above approximately $900 \mathrm{~K} . \mathrm{N}_{S}$ is an effective trap for vacancies, which are mobile above these temperatures..$^{15}$ Charge transfer from $\mathrm{N}_{S}^{0}$, which is a deep donor, creates $\mathrm{NV}^{-}$, and both the neutral and negative charge states of the NV center are routinely detected by photoluminescence in the same diamond. ${ }^{16,17} \mathrm{NV}$ centers can be created at specific locations by $\mathrm{N}^{+}$or $\mathrm{N}_{2}^{+}$ion implantation. ${ }^{18} \mathrm{NV}$ centers are also found in as-grown diamond synthesized by chemical vapor deposition (CVD) ${ }^{16}$ although it has not been established if the defect is grown in as a unit or is formed by $\mathrm{N}_{S}$ trapping a vacancy, which is mobile at typical growth temperatures. High pressure high-temperature (HPHT) treatment of brown natural diamond to improve the color can also produce $\mathrm{NV}^{-}$centers. ${ }^{19}$ Vacancy clusters are thought to be the cause of the brown color; ${ }^{20} \mathrm{NV}$ centers are formed when vacancies, released by the HPHT annealing, are trapped at $\mathrm{N}_{S}$ which can be produced by the dissociation of nitrogen aggregates.

Three synthetic single-crystal diamonds were used in this study, labeled A-C. NV centers were created in all three samples through electron irradiation and subsequent annealing at $1100 \mathrm{~K}$; the annealing temperature was chosen so as to anneal out all isolated vacancies, as shown by, e.g., Davies and Hamer. ${ }^{7}$ To study the hyperfine interaction with the two different nitrogen isotopes, samples were prepared either containing the natural abundance of nitrogen isotopes $\left({ }^{14} \mathrm{~N}\right.$ rich, sample A) or enriched with ${ }^{15} \mathrm{~N}$. The ${ }^{15} \mathrm{~N}$ enrichment can be achieved through either HPHT (sample B) or CVD (sample C) synthesis. A summary of sample preparation and properties is given in Table I.

Sample A was grown by conventional HPHT synthesis using a cobalt-containing solvent catalyst with the addition of titanium as a nitrogen getter. The remaining nitrogen was made up of the natural abundance of isotopes $\left(99.6 \%{ }^{14} \mathrm{~N}\right)$. The sample was cut predominately from a cube growth sector although it contains small volumes of other growth sectors. Infrared measurements of the as-grown sample showed it to contain 9(1) ppm (parts per million carbon atoms) $\mathrm{N}_{S}^{0}$. The sample was irradiated with $1.9 \mathrm{MeV}$ electrons to a dose of $3 \times 10^{18} \mathrm{e} / \mathrm{cm}^{2}$, yielding a vacancy concentration ${ }^{21}$ of $\sim 25 \mathrm{ppm}$. The sample was subsequently annealed at $1100 \mathrm{~K}$ in a nonoxidizing atmosphere for $4 \mathrm{~h}$.

Sample B was synthesized using a modified HPHT process: $^{22}$ the growth capsule with the carbon source and 
a)

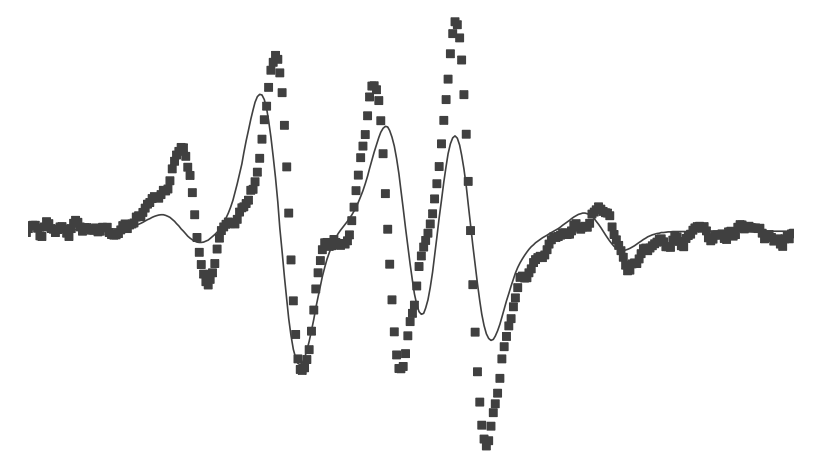

$\begin{array}{cccccc}164.7 & 164.8 & 164.9 & 165.0 & 165.1 & 165.2 \\ & & & \\ & \text { Magnetic Field (mT) }\end{array}$

b)

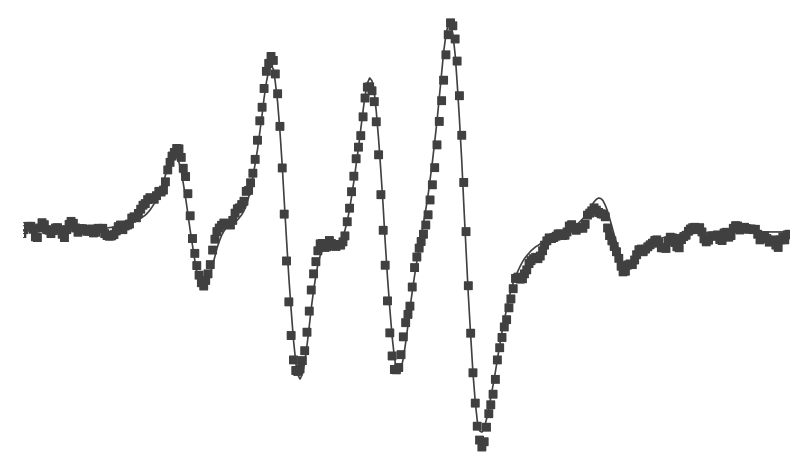

FIG. 2. First harmonic EPR spectra of the half-field, $M_{S}=-1 \rightarrow 1$, transitions for the ${ }^{14} \mathrm{NV}^{-}$defect with the magnetic field $\mathbf{B}$ applied along $\langle 110\rangle$. The squares show the experimental data points recorded for the ${ }^{14} \mathrm{~N}$-doped sample $\mathrm{A}$ and the lines show the simulated spectrum using (a) the parameters from He et al. (Ref. 24) and (b) the parameters determined in this study. The spectrum is complicated due to the similar magnitudes of the nitrogen hyperfine, nuclear Zeeman, and quadrupole interactions, which results in considerable nuclear spin-state mixing such that $m_{I}$ is no longer a good quantum number.

solvent/catalyst reaction mixture was pretreated at high temperature in vacuum to remove the vast majority of the atmospheric gases. The removed gas was replaced with ${ }^{15} \mathrm{~N}$ enriched gas at a reduced temperature and the capsule was sealed. The reaction mixture was then subjected to HPHT conditions in the diamond stable region producing material with $\sim 150 \mathrm{ppm}_{S}^{0}$, as measured using EPR. The sample was HPHT annealed leading to $>80 \%$ aggregation of the nitrogen to $A$ centers (nitrogen pairs) and then irradiated with 1.5 MeV electrons to a dose of $4 \times 10^{17} \mathrm{e} / \mathrm{cm}^{2}$, leading to a vacancy concentration ${ }^{21}$ of $\sim 3 \mathrm{ppm}$. (This sample contains different growth sectors with differing $\mathrm{N}_{S}$ concentrations so that in some sectors the vacancy concentration was of the same order of magnitude as the $\mathrm{N}_{S}$ concentration.) After the irradiation the sample was annealed in a nonoxidizing atmosphere at $1100 \mathrm{~K}$ for $4 \mathrm{~h}$.

Sample C was homoepitaxially grown on a $\{100\}$ substrate using CVD synthesis. ${ }^{15} \mathrm{~N}$ enriched $\mathrm{N}_{2}$ was added to the CVD source gases and the nitrogen-doped CVD sample produced had $\sim 90 \%{ }^{15} \mathrm{~N}$ enrichment. Optical absorption measurements showed that as grown the sample contained approximately $2 \mathrm{ppm} \mathrm{N}_{S}^{0}$. The sample was irradiated with $1.5 \mathrm{MeV}$ electrons to a dose of $6 \times 10^{17} \mathrm{e} / \mathrm{cm}^{2}$, yielding a vacancy concentration $^{21}$ of $\sim 5 \mathrm{ppm}$ and subsequently annealed at $1100 \mathrm{~K}$ in a nonoxidizing atmosphere.

EPR measurements were made using a Bruker EMX spectrometer operating at $\sim 9.6 \mathrm{GHz}$. The EPR linewidth for the $\mathrm{NV}^{-}$spectrum was $\sim 0.02 \mathrm{mT}$ in all three samples. The spectrometer was equipped with an Oxford Instruments Liquid Helium ESR900 flow cryostat allowing measurements in the temperature range of 4-300 K. For optical excitation the output from a $200 \mathrm{~W} \mathrm{Hg}-\mathrm{Xe}$ arc lamp was focused onto the sample in the EPR cavity through a liquid light guide transmitting light of wavelengths between 300 and $700 \mathrm{~nm}$. The system for delivering the light to the sample has previously been measured to have a transmission of $\sim 20 \% .^{23}$ The energy spectrum of the optical excitation was further controlled with the use of low-pass filters.

\section{RESULTS}

To study the nitrogen hyperfine interaction of $\mathrm{NV}^{-}, \mathrm{EPR}$ spectra for the half-field "forbidden" $\left(\Delta M_{S}= \pm 2\right)$ transitions were recorded for all samples in the dark with the applied magnetic field, $\mathbf{B}$, parallel to the crystallographic directions $\langle 001\rangle,\langle 110\rangle$, and $\langle 111\rangle$, to better than $0.05^{\circ}$. The half-field spectra were used since to first order the relative positions of these are independent of the large zero-field splitting which makes the full-field $\left(\Delta M_{S}= \pm 1\right)$ lines move by up to $2.5 \mathrm{mT}$ per degree of misalignment; consequently the half-field lines are less sensitive to slight misorientations of the field with respect to the chosen crystallographic direction. Figure 2 shows a room-temperature EPR spectrum for sample A recorded with $\mathbf{B}$ parallel to $\langle 110\rangle$. This spectrum does not show the characteristic three line pattern of hyperfine interaction with one $I=1$ nucleus such as ${ }^{14} \mathrm{~N}$. The similar magnitudes of the nitrogen hyperfine, nuclear Zeeman, and quadrupole interactions result in considerable nuclear spin-state mixing, such that $m_{I}$ is no longer a good quantum number and EPR transition probabilities must be calculated for all possible transitions between spin states. Changing the nitrogen isotope from ${ }^{14} \mathrm{~N}$ to ${ }^{15} \mathrm{~N}$ removes the quadrupole interaction simplifying the spectrum considerably, as can be seen in Fig. 3, which shows the EPR spectra recorded with the magnetic field applied parallel to $\langle 001\rangle$ for (a) the ${ }^{14} \mathrm{~N}$-doped sample $\mathrm{A}$ and (b) the ${ }^{15} \mathrm{~N}$-doped sample $\mathrm{C}$. In the latter case only a small forbidden transition is visible in the center of the spectrum.

Optically excited EPR spectra were also recorded for all samples at temperatures between 4 and $300 \mathrm{~K}$. The optical excitation increased the intensity of the $\mathrm{NV}^{-}$spectrum by more than two orders of magnitude at room temperature, 
a)

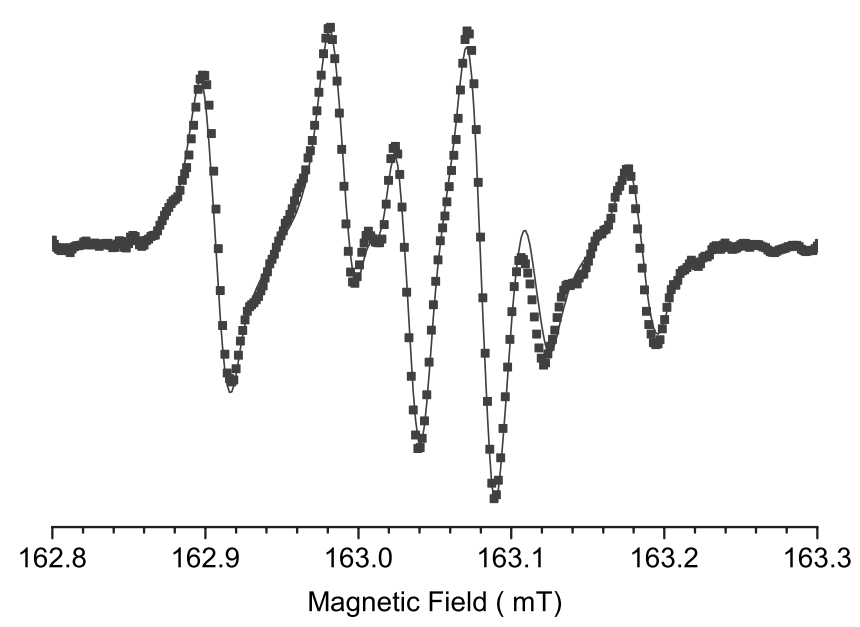

b)

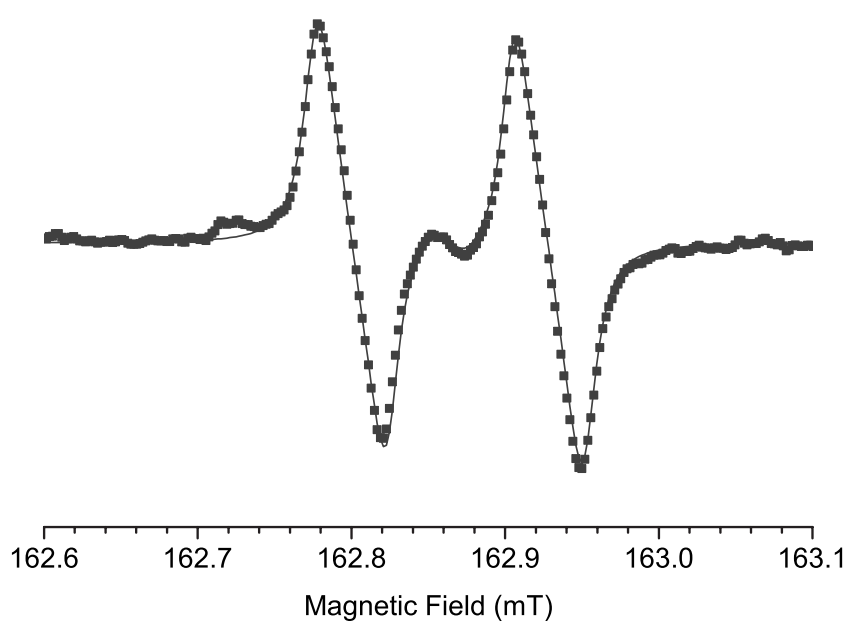

FIG. 3. First harmonic EPR spectra of the half-field, $M_{S}=-1 \rightarrow 1$, transitions with the magnetic field $\mathbf{B}$ applied along $\langle 001\rangle$ for (a) ${ }^{14} \mathrm{NV}^{-}$ and (b) ${ }^{15} \mathrm{NV}^{-}$. The squares represent the experimental data points measured in (a) sample A and (b) sample C. The lines show the simulated spectrum using the hyperfine parameters determined in this study. The experimental spectrum in (b) is modulation broadened, which is accounted for in the fit through pseudomodulation. Note how the confusion of lines due to spin-state mixing resulting from the similarity in magnitude of the ${ }^{14} \mathrm{~N}$ hyperfine, nuclear Zeeman, and quadrupole interactions in (a) simplifies to the two line spectrum of (b) with clear $m_{I}=\frac{1}{2}$ lower field and $m_{I}=-\frac{1}{2}$ higher field lines when ${ }^{14} \mathrm{~N}$ is substituted with ${ }^{15} \mathrm{~N}$.

allowing the satellite lines due to hyperfine interaction with the $1.1 \%$-naturally abundant ${ }^{13} \mathrm{C}$ atoms in the samples to be easily detected (see Fig. 4). These measurements were also used to study the ensemble spin polarization of the $\mathrm{NV}^{-}$ center at different temperatures.

\section{ANALYSIS OF THE DATA}

The EPR spectra from the $\mathrm{NV}^{-}$defect were fitted to the spin Hamiltonian

$$
\begin{aligned}
H= & \mu_{B} \mathbf{B} \cdot \underline{\mathbf{g}} \cdot \mathbf{S}+\mathbf{S} \cdot \underline{\mathbf{D}} \cdot \mathbf{S} \\
& +\sum_{j}\left(\mathbf{S} \cdot \underline{\mathbf{A}}_{j} \cdot \mathbf{I}_{j}+\mathbf{I}_{j} \cdot \underline{\mathbf{P}}_{j} \cdot \mathbf{I}_{j}-g_{N_{j}} \mu_{N} \mathbf{B} \cdot \mathbf{I}_{j}\right),
\end{aligned}
$$

where all the terms have their usual meaning. In fitting the experimental data from all three samples, we found values for the electronic Zeeman interaction (g) and zero-field splitting (D) that agree with what has been reported previously. ${ }^{1,24}$ Our value for the zero-field splitting, $D_{\|}=\frac{3}{2} D_{z}=2.872(2) \mathrm{GHz}$ is within experimental error identical to Loubser and van Wyk's ${ }^{1} D_{\|}=2.878(6) \mathrm{GHz}$. However, a smaller experimental error in our determined $\mathbf{g}$ allows us to discern a small anisotropic component of the electronic Zeeman interaction, yielding $g_{\|}=2.0029(2)$ and $g_{\perp}=2.0031(2)$, where $g_{\|}$lies along the $\langle 111\rangle \mathrm{NV}$ symmetry axis. The nitrogen hyperfine $(\underline{\mathbf{A}})$ and quadrupole $(\underline{\mathbf{P}})$ interactions were constrained to be axially symmetric around the $C_{3 v}$ symmetry axis of the defect; relaxing this constraint did not lead to a statistically significant improvement in the quality of the fit. The previously determined ${ }^{14} \mathrm{~N}$ hyperfine and quadrupole parameters $^{24}$ could not satisfactorily reproduce the experimental data; an example of the resulting discrepancy between simulation and experiment is shown for a spectrum recorded with $\mathbf{B} \|\langle 110\rangle$ in Fig. 2(a). The parameters deter- mined by fitting the reported data are given in Table II, where the ${ }^{14} \mathrm{~N}$ parameters were fitted to spectra from sample $\mathrm{A}$, and the ${ }^{15} \mathrm{~N}$ parameters were fitted to spectra from samples B and C. Figure 2(b) shows an experimental EPR spectrum for sample A with $\mathbf{B} \|\langle 110\rangle$ together with a simulated spectrum using the hyperfine parameters determined in this study. The new parameters yield an excellent fit to the

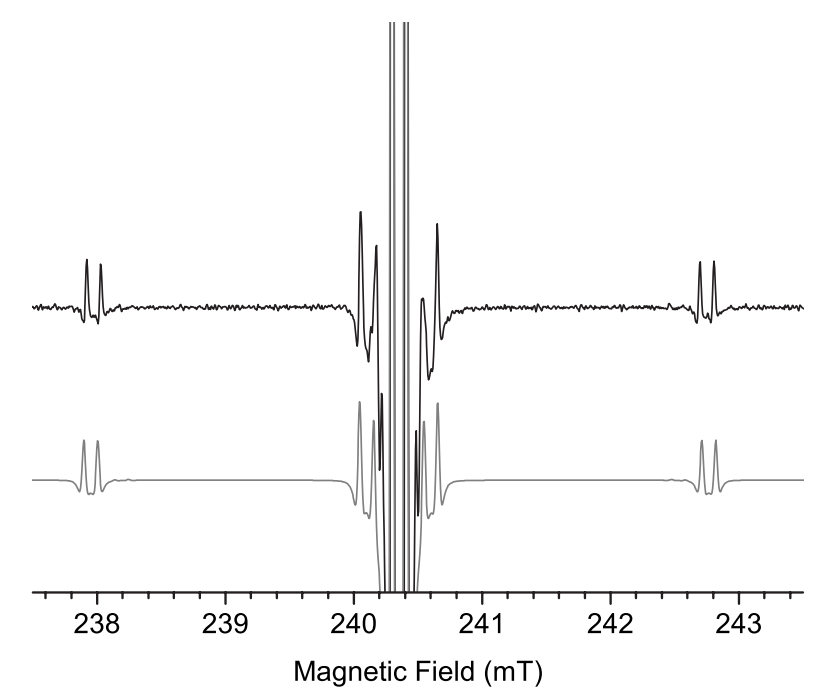

FIG. 4. Optically excited EPR spectrum from the ${ }^{15} \mathrm{~N}$-doped sample B recorded with the magnetic field $\mathbf{B}$ applied along $\langle 111\rangle$, showing the $M_{S}=0 \rightarrow 1$ transitions for the defects with their axes parallel to the magnetic field. The upper black line shows the second-harmonic experimental spectrum and the lower grey line shows the simulated spectrum using the spin-Hamiltonian parameters from Tables II and III. The intensities of the ${ }^{13} \mathrm{C}$ satellites in the simulated spectrum correspond to three and six equivalent $\mathrm{C}$-atom positions. 
TABLE II. Nitrogen hyperfine parameters for the $\mathrm{NV}^{-}$defect determined at room temperature. For the ${ }^{14} \mathrm{NV}^{-}$the quadrupole parameter is also given. All interactions are axially symmetric about the NV symmetry axis, shown as [111] in Fig. 1 . The ${ }^{14} \mathrm{~N}$ parameters were fitted to spectra from sample $\mathrm{A}$, and the ${ }^{15} \mathrm{~N}$ parameters were fitted to spectra from samples B and C.

\begin{tabular}{llll}
\hline \hline Defect & $A_{\|} / \mathrm{MHz}$ & $A_{\perp} / \mathrm{MHz}$ & $P_{\|} / \mathrm{MHz}$ \\
\hline${ }^{14} \mathrm{NV}^{-}$ & $-2.14(7)$ & $-2.70(7)$ & $-5.01(6)$ \\
${ }^{14} \mathrm{NV}^{-}\left(\mathrm{He}\right.$ et al. $\left.{ }^{\mathrm{a}}\right)$ & $+2.30(2)$ & $+2.10(10)$ & $-5.04(5)$ \\
${ }^{15} \mathrm{NV}^{-}$ & $+3.03(3)$ & $+3.65(3)$ & \\
\hline \hline
\end{tabular}

Reference 24 .

experimental data for all samples and magnetic field directions, as is further demonstrated in Fig. 3.

Hyperfine satellites from two sets of ${ }^{13} \mathrm{C}$ atoms were detected (see Fig. 4). These were fitted (separately) to Eq. (1), varying only the ${ }^{13} \mathrm{C}$ hyperfine interaction. The hyperfine interaction was constrained to have two principal axes in a $\{110\}$ plane not containing the trigonal axis of the NV defect (see Table III). Removing the constraints does not improve the fit. Both the larger ${ }^{13} \mathrm{C}$ hyperfine interaction, labeled $\mathrm{C}_{a}$, and the smaller ${ }^{13} \mathrm{C}$ hyperfine interaction, labeled $\mathrm{C}_{g}$, are axially symmetric with $A_{\|}$along a crystallographic $\langle 111\rangle$ direction which is not the symmetry axis of the $\mathrm{NV}$ center. The experimentally recorded intensity for the $\mathrm{C}_{a}$ and $\mathrm{C}_{g}$ hyperfine satellites are reproduced assuming they are due to $3.0(1)$ and 6(1) equivalent ${ }^{13} \mathrm{C}$ neighbors, respectively (see Fig. 4). The ${ }^{13} \mathrm{C}_{a}$ hyperfine interaction is almost independent of the temperature in the range of $10-300 \mathrm{~K}$ measured here and the specific nitrogen isotope, ${ }^{14} \mathrm{~N}$ or ${ }^{15} \mathrm{~N}$, involved in the $\mathrm{NV}^{-}$ center (see Table III).

The intensity of an EPR line is proportional to the difference in population of the two states between which the transition occurs. In the absence of spin selective excitations, this population difference between states split by the interactions described in Eq. (1) is determined by Boltzmann statistics. For $\mathrm{NV}^{-}$under conditions of optical excitation, this may not be the case, depending on the energy of the light. Harrison et $a l .{ }^{12}$ have previously shown that optical excitation populates the $M_{S}=0$ level favorably compared to the $M_{S}= \pm 1$ levels. The spin polarization of $\mathrm{NV}^{-}$, defined as the relative population of the $M_{S}=0$ level, can be calculated from the ratio $\zeta$

TABLE III. ${ }^{13} \mathrm{C}$ hyperfine parameters for the $\mathrm{NV}^{-}$defect with directions $[\theta, \varphi]$, where $\theta$ is the angle from the crystallographic [001] direction and $\varphi$ is the angle from [100] measured toward [010] in the (100) plane. The ${ }^{14} \mathrm{~N}$ parameters were fitted to spectra from sample A, and the ${ }^{15} \mathrm{~N}$ parameters were fitted to spectra from samples B and C.

\begin{tabular}{ccc}
\hline \hline Defect & $A_{\|} / \mathrm{MHz}$ & $A_{\perp} / \mathrm{MHz}$ \\
\hline${ }^{14} \mathrm{NV}^{-13} \mathrm{C}_{\mathrm{a}}$ at $10 \mathrm{~K}$ & $199.7(2) \|\left[125.26(3)^{\circ}, 45^{\circ}\right]$ & $120.3(2)$ \\
${ }^{15} \mathrm{NV}^{-13} \mathrm{C}_{\mathrm{a}}$ at $10 \mathrm{~K}$ & $199.1(1) \|\left[125.26(2)^{\circ}, 45^{\circ}\right]$ & $121.1(1)$ \\
${ }^{15} \mathrm{NV}^{-13} \mathrm{C}_{\mathrm{a}}$ & & \\
at room temperature & $198.2(3) \|\left[125.26(5)^{\circ}, 45^{\circ}\right]$ & $120.8(2)$ \\
${ }^{15} \mathrm{NV}^{-13} \mathrm{C}_{\mathrm{g}}$ at $10 \mathrm{~K}$ & $18.49(5) \|\left[125.3(1)^{\circ}, 45^{\circ}\right]$ & $13.26(5)$ \\
\hline \hline
\end{tabular}

of the EPR intensity measured in the dark and the EPR intensity measured with optical excitation if some assumption is made about the relative populations of the $M_{S}= \pm 1$ levels We assume that the population is equal in the two $M_{S}= \pm 1$ levels, i.e., that the optical pumping is fast compared to the spin-lattice relaxation. This is a reasonable assumption at low temperatures where the spin-lattice relaxation times are long (Harrison et al. ${ }^{25}$ determined the spin-lattice relaxation time for the $\mathrm{NV}^{-}$center to be 28 and $265 \mathrm{~s}$ in two different samples at $2 \mathrm{~K}$ ). At high temperatures where the difference in population of the spin levels is small, this assumption is roughly equivalent to assuming that population is lost equally from the $M_{S}= \pm 1$ levels. When sample B was illuminated with the full output of the lamp (300-700 nm) at room temperature $(\sim 300 \mathrm{~K})$, $\zeta$ was found to be 242 and -244 , respectively, for the $M_{S}=0 \rightarrow 1$ and $M_{S}=-1 \rightarrow 0$ transitions from the $\mathrm{NV}^{-}$defects with their $C_{3 v}$ axes parallel with B. Both these ratios yield a spin polarization at room temperature of $\sim 42(4) \%$, confirming the validity of the assumption that both $M_{S}= \pm 1$ levels are equally populated. Corresponding measurements were also made at 100 and $10 \mathrm{~K}$. For the measurements in the dark microwave power saturation was unavoidable so that $\zeta$ could not be determined directly for these temperatures. However, the spin polarization can still be determined by comparison with the dark measurement at room temperature, assuming Boltzmann statistics, and is found to be $62(6) \%$ at $100 \mathrm{~K}$ and $65(6) \%$ at $10 \mathrm{~K}$, where again the intensities for both the $M_{S}=0 \rightarrow 1$ and $M_{S}=-1 \rightarrow 0$ transitions yield the same spin-polarization value. Samples A and $\mathrm{C}$ show a smaller degree of spin polarization, 38(4)\% and 35(4)\% at room temperature, $48(5) \%$ and $45(5) \%$ at $100 \mathrm{~K}$, and $52(5) \%$ and $51(5) \%$ at $10 \mathrm{~K}$, respectively. As for sample $\mathrm{B}$, the same degree of spin polarization is found for both the $M_{S}=0 \rightarrow 1$ and $M_{S}=-1 \rightarrow 0$ transitions at all temperatures.

\section{DISCUSSION}

\section{A. Quadrupole interaction}

Tucker et $a l .{ }^{26}$ showed that, for defects in diamond containing substitutional nitrogen, the ${ }^{14} \mathrm{~N}$ quadrupole parameter is a measure of the unpaired-electron population in a $2 p$ orbital on the nitrogen atom and takes values in the range $-2>P_{\|}>-5.5 \mathrm{MHz}$. The value for $\mathrm{NV}^{-}$of $P_{\|}=-5.01 \mathrm{MHz}$ implies that less than $2 \%$ of the unpairedelectron probability density is found in the $2 p$ orbital on the nitrogen atom. This supports the conclusion that the unpaired-electron probability density is mainly located on the dangling orbitals from the three $\mathrm{C}$ atoms neighboring the vacancy.

\section{B. Zero-field splitting}

Harrison et al. ${ }^{12}$ have experimentally determined the sign of the zero-field splitting to be positive from depopulation studies at low temperatures, $T>2 \mathrm{~K}$. This measurement is inherently sensitive to problems with microwave power saturation. The relative signs of the zero-field, quadrupole, and hyperfine interactions can be determined from the positions and intensities of the electron-nuclear double spin-flip tran- 
TABLE IV. Isotropic $(a)$ and anisotropic $(b)$ components of the hyperfine interaction for $\mathrm{NV}^{-}$centers. The experimental values are calculated from the hyperfine parameters given in Table II. The density functional theory hyperfine parameters are from the study by Gali et al. (Ref. 5); the value for ${ }^{15} \mathrm{~N}$ was converted from the ${ }^{14} \mathrm{~N}$ parameter assuming that the hyperfine interaction scales with the ratio of the nuclear Zeeman parameters (Ref. 28). Details of the extended point-dipole calculation are given in the text.

\begin{tabular}{|c|c|c|c|c|c|}
\hline \multirow[b]{2}{*}{ Defect } & \multicolumn{2}{|c|}{ Experimental } & \multicolumn{2}{|c|}{ Density-functional theory ${ }^{a}$} & \multirow{2}{*}{$\begin{array}{c}\begin{array}{c}\text { Extended point } \\
\text { dipole }\end{array} \\
b / \mathrm{MHz}\end{array}$} \\
\hline & $a / \mathrm{MHz}$ & $b / \mathrm{MHz}$ & $a / \mathrm{MHz}$ & $b / \mathrm{MHz}$ & \\
\hline${ }^{14} \mathrm{NV}^{-}$ & $-2.51(7)$ & $0.19(7)$ & -1.7 & $\sim 0$ & 0.18 \\
\hline${ }^{15} \mathrm{NV}^{-}$ & $3.44(3)$ & $-0.21(3)$ & 2.4 & $\sim 0$ & -0.25 \\
\hline
\end{tabular}

${ }^{\mathrm{a}}$ Reference 5 .

sitions $\left(\Delta M_{S}= \pm 1, \Delta m_{I}= \pm 1\right)$. An alternative determination of the sign of $D$ can therefore be made by noting that for defects with a substitutional nitrogen the ${ }^{14} \mathrm{~N}$ quadrupole interaction has a negative sign. ${ }^{26}$ Fitting to the experimental data then constrains the sign of the zero-field splitting to be positive. This is the opposite sign expected when the dipoledipole interaction is the dominant contribution to the zerofield splitting. The maximum deviation of the electronic Zeeman interaction from the free-electron value $\left(g_{e}=2.0023\right)$ is $\Delta g=0.0008$; assuming a spin-orbit coupling parameter $\lambda_{\mathrm{so}} \sim 4.5 \times 10^{5} \mathrm{MHz}$ which is the value given for an $S=1$ electron system on a free $\mathrm{C}$ atom by Gerloch, ${ }^{27}$ this leads to a contribution to $D$ from spin-orbit coupling of $\sim 270 \mathrm{MHz}$. This is an order-of-magnitude smaller than the experimentally determined $D$ of $2.872(2) \mathrm{GHz}$. We therefore conclude that the zero-field splitting in the $\mathrm{NV}^{-}$center is due to anisotropic exchange. Determining the anisotropic exchange interaction is a taxing theoretical problem. Reanalysis of Loubser and van Wyk's ${ }^{1}$ data on the decrease in EPR line intensity at temperatures between 300 and $550 \mathrm{~K}$ allows the isotropic exchange with the low lying excited state to be determined as $\sim 27 \mathrm{THz}(\sim 0.1 \mathrm{eV})$. The anisotropy of the exchange interaction therefore only needs to be of the order of $0.01 \%$ to account for the zero-field splitting and is consistent with the low lying ${ }^{1} A$ state predicted by Gali et al. ${ }^{5}$

\section{Nitrogen hyperfine interaction}

Given the negative sign for the quadrupole interaction, we find from the position and intensities of the electron-nuclear double spin-flip transitions that the sign of the hyperfine interaction is negative for ${ }^{14} \mathrm{~N}$; we therefore assume a positive sign of the hyperfine interaction for ${ }^{15} \mathrm{~N}$ (see Table II) since $g_{\mathrm{N}}$ for ${ }^{14} \mathrm{~N}$ is 0.4038 and $g_{\mathrm{N}}$ for ${ }^{15} \mathrm{~N}$ is $-0.5664 .{ }^{28}$ This result is in contradiction to $\mathrm{He}$ et al. $^{24}$ who argued that since the nuclear $g$ factor for ${ }^{14} \mathrm{~N}$ is positive then the hyperfine interaction should be, as well. Figure 2 shows a comparison of the experimental spectrum for the ${ }^{14} \mathrm{~N}$-doped sample A, with simulated spectra using the nitrogen hyperfine parameters of $\mathrm{He}$ et al. $^{24}$ in (a) and the parameter determined in this study in (b). Only the new parameters yield a satisfactory fit. The fit is equally good for all samples and magnetic field orientations studied (see also Fig. 3). A large part of the discrepancy between the simulated spectra based on the parameters of He et al. ${ }^{24}$ and the experimental spectra is due to a difference in transition probabilities for the EPR lines. This problem would not have been evident to He et al. ${ }^{24}$ since they determined their hyperfine parameters from continuous-wave electron-nuclear double resonance (ENDOR) measurements, which do not yield quantitative information about transition probabilities. Gali et al. ${ }^{5}$ also found a negative sign for the isotropic component of the ${ }^{14} \mathrm{~N}$ hyperfine interaction from DFT calculations. The calculated nitrogen hyperfine interaction is isotropic and smaller than what is found experimentally. However, Gali et al. ${ }^{5}$ explicitly state that there is an inherent error of about $0.3 \mathrm{MHz}$ in the calculated anisotropic term, which is smaller than the experimentally determined anisotropic component (see Table IV). Therefore the theoretical nitrogen hyperfine parameters are in reasonable agreement with the experimental values.

Table IV shows the isotropic $(a)$ and anisotropic $(b)$ components of the nitrogen hyperfine interaction. The experimental parameters have been calculated from the parameters given in Table II as $a=\left(A_{\|}+2 A_{\perp}\right) / 3$ and $b=\left(A_{\|}-A_{\perp}\right) / 3$. The fact that the isotropic hyperfine component is small and of opposite sign to that expected for localization of the unpaired-electron probability density on the nitrogen nucleus indicates that this contribution arises through spin polarization. The unpaired-electron probability density is predominately localized on the three carbon neighbors (this ${ }^{3} \mathrm{~A}_{2}$ state does not involve orbitals related to nitrogen). This polarizes the core states of the nitrogen and, since the nuclear magneton for ${ }^{14} \mathrm{~N}$ is positive, the Fermi contact term will be negative. The small anisotropic hyperfine component can be explained by dipolar interaction between the unpaired-electron probability density and the nitrogen nucleus, calculated as follows: One third of the unpaired-electron probability density was assumed to be localized at each of the carbon atoms neighboring the vacancy. The dipolar interaction between the nitrogen nucleus and an electron localized $2.51 \AA$ away (next-nearest-neighbor distance in the diamond lattice) was calculated along the three different directions. These three contributions were transformed to a common coordinate axis system and summed. The calculated anisotropic hyperfine component was found by diagonalizing the resulting hyperfine matrix and is shown in Table IV, labeled as "extended point dipole." Given the approximation inherent in this simple model, the agreement between the experimental and calculated values is fortuitous. The small value of $b$ in this 


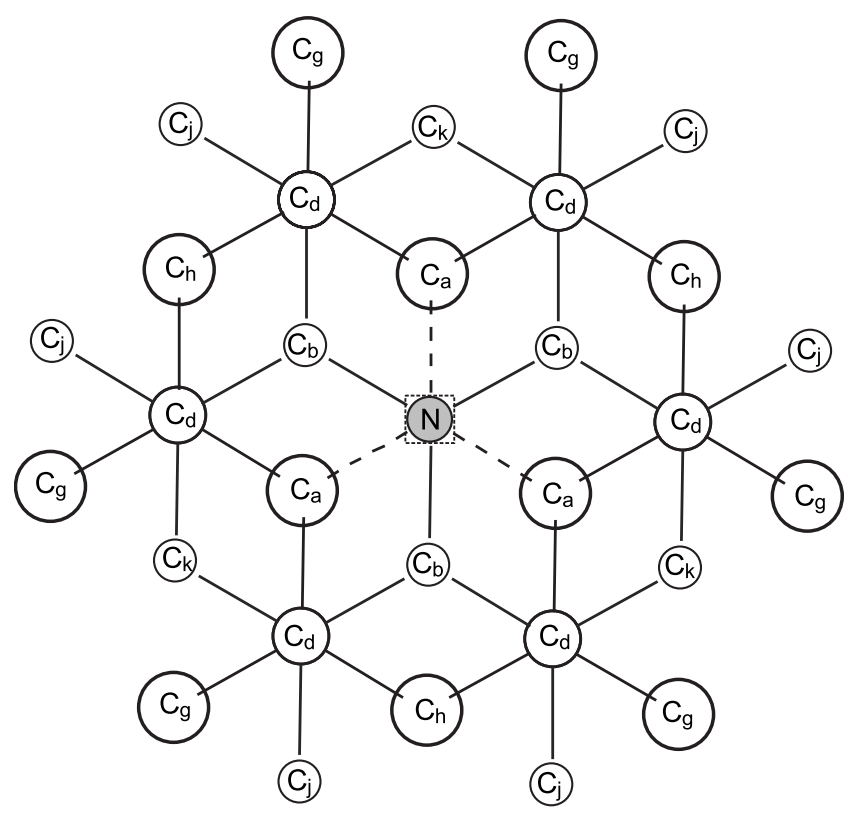

FIG. 5. Projection of the nitrogen vacancy center in diamond on the (111) plane, showing equivalent sets of $\mathrm{C}$ neighbors. The labeling system is the same as that used in Fig. 1.

calculation is due to partial cancellation of the three contributions, and is very sensitive to relaxation of the atom positions and further delocalization of the unpaired-electron probability density. For example, assuming $28 \%$ of the unpaired-electron probability density is localized on each of the three carbon neighbors (see Sec. V D) changes $b$ for ${ }^{14} \mathrm{~N}$ to $0.15 \mathrm{MHz}$. The value of $b$ can be returned to $0.18 \mathrm{MHz}$ by shifting the carbon atoms by $0.2 \AA$ toward the vacancy. Therefore too much should not be read into the numerical agreement but the calculation shows that the model can provide a satisfactory explanation for the small value of $b$.

\section{D. ${ }^{13} \mathrm{C}$ hyperfine interaction}

The relative intensity of the ${ }^{13} \mathrm{C}$ satellites to the appropriate central transition is reproduced by assuming there are $3 \pm 0.1$ equivalent positions for the larger hyperfine interaction, ${ }^{13} \mathrm{C}_{a}$, and $6 \pm 1$ equivalent positions for the smaller hyperfine interaction, ${ }^{13} \mathrm{C}_{g}$. Considering the directions of the principal values of the hyperfine interactions and number of equivalent positions for the $\mathrm{C}$ atoms, the larger one is assigned to the three carbon atoms neighboring the vacancy, i.e., the atoms labeled $\mathrm{C}_{a}$ in Figs. 1 and 5. This assignment is supported by recent DFT calculations predicting hyperfine parameters of $A_{\|}=185.4 \mathrm{MHz}$ and $A_{\perp}=109.9 \mathrm{MHz}$ which are in reasonable agreement with the experimental values for the $\mathrm{C}_{a}$ atoms (see Table III). The parameters for the $\mathrm{C}_{a}$ are in good agreement with the ${ }^{13} \mathrm{C}$ hyperfine interaction reported by Loubser and van Wyk. ${ }^{1}$

Loubser and van $\mathrm{Wyk}^{29}$ found a second ${ }^{13} \mathrm{C}$ hyperfine interaction, which they report as isotropic with a magnitude of $15 \mathrm{MHz}$ and due to three equivalent atoms. In contrast, we find the second set of ${ }^{13} \mathrm{C}$ hyperfine parameters to be anisotropic and due to six equivalent atoms. However, we also find the isotropic component to be $15 \mathrm{MHz}$ and the aniso- tropic component is only $1.74 \mathrm{MHz}$. Gali et al. ${ }^{5}$ predict a hyperfine interaction of $A_{\|}=19.4 \mathrm{MHz}$ and $A_{\perp}=13.9 \mathrm{MHz}$ with six equivalent carbon atoms at the third neighbor distance $\left(\mathrm{C}_{g}\right)$, which agrees well with our experimental findings (see Table III). The six nearest neighbors to the $\mathrm{C}$ atoms around the vacancy, with their bonds not parallel to the NV axis, i.e., the atoms labeled $\mathrm{C}_{d}$ in Figs. 1 and 5 might from pure geometrical considerations be assumed to give rise to a larger hyperfine interaction since they are closer to the dangling bonds of the vacancy where the majority of the unpaired-electron probability density is localized. However, Gali et al.' $\mathrm{s}^{5}$ calculations suggest that the hyperfine interaction with these atoms is less than $5 \mathrm{MHz}$ and therefore too small to be resolved from the main $\mathrm{NV}^{-}$EPR lines. Presumably the hyperfine interaction is small due to partial cancellation of direct and indirect (e.g., spin-spin polarization) contributions.

According to the DFT calculations by Gali et al., ${ }^{5}$ the third largest ${ }^{13} \mathrm{C}$ hyperfine interaction is with a set of three carbon atoms again at the third neighbor distance (atoms $\mathrm{C}_{l}$ in Fig. 1). This hyperfine interaction is predicted to have $A_{\|}=18.0 \mathrm{MHz}$ and $A_{\perp}=12.8 \mathrm{MHz}$. We do not detect these satellites in our experiments. As can be seen from Fig. 4, the satellites due to the smaller ${ }^{13} \mathrm{C}$ hyperfine interaction, ${ }^{13} \mathrm{C}_{g}$, are only just resolved from the main $\mathrm{NV}^{-}$lines. It is therefore possible that the hyperfine interaction with this second set of three carbon atoms is simply too small to be resolved from the main line.

Interpreting the ${ }^{13} \mathrm{C}$ hyperfine parameters in the usual manner, ${ }^{30}$ it is found that the two sets of carbon neighbors account for $\sim 96 \%$ of the unpaired-electron probability density for $\mathrm{NV}^{-}$, with $\sim 84 \%$ on the three carbons nearest the vacancy, consistent with theoretical predictions.

\section{E. Spin polarization}

The dependence of the $\mathrm{NV}^{-}$EPR intensity on the lamp power was measured to check if the determined spinpolarization values are illumination intensity limited. Decreasing the lamp power by $25 \%$ from 200 to $150 \mathrm{~W}$, and therefore the power of the light arriving at the sample from 400 to $300 \mathrm{~mW}$, only decreases the spin polarization in sample B from $42 \%$ to $40 \%$, which indicates that the limiting factor for the spin polarization may not be the lamp power but rather a more fundamental property of the $\mathrm{NV}^{-}$defect. In our experiments the ensemble spin polarization of $\mathrm{NV}^{-}$increases from $\sim 42 \%$ at room temperature to $\sim 62 \%$ at $100 \mathrm{~K}$ in sample B, which shows the largest degree of spin polarization. Decreasing the temperature further to $10 \mathrm{~K}$ does not give a significant increase in spin polarization for any of the samples. The spin polarization found in this study is significantly lower than the values reported by Harrison et al. ${ }^{25}$ They only measure the signal intensity for the $M_{S}=-1 \rightarrow 0$ transition and in their calculations they assume that unpaired-electron probability density is lost equally from the $M_{S}= \pm 1$ levels, an assumption that cannot be justified at low temperature. Reanalyzing their data using the assumption made in this paper that the $M_{S}= \pm 1$ levels are equally populated, we find a spin polarization of $\sim 80 \%$. A further possible source of error in the data of Harrison et al. ${ }^{25}$ could be 
the fact that they referenced to measurements under dark conditions which may be affected by microwave power saturation especially considering the low temperatures $(<2 \mathrm{~K})$ used. This would lead to an overestimate of the degree of spin polarization.

The samples studied here were selected and processed to facilitate ensemble measurements on the $\mathrm{NV}^{-}$center; the samples and processing conditions were not optimized for the production of isolated $\mathrm{NV}^{-}$centers unperturbed by other defects, strain, etc. The most promising results for quantum computation, where $\sim 100 \%$ spin polarization has been achieved, are obtained from synthetic diamond produced with nitrogen doping at much lower levels.

\section{CONCLUSIONS}

We have redetermined values of the nitrogen hyperfine interaction in the $\mathrm{NV}^{-}$center, reversing the sign of this interaction from the values in literature, ${ }^{24}$ in agreement with recent DFT calculations. ${ }^{5}$ A simple calculation of dipolar interaction between the nitrogen nucleus and the unpairedelectron probability density localized on the nearest-neighbor carbon atoms yields good agreement with the experimental values of the anisotropic hyperfine interaction. This assumption that the unpaired-electron probability density is mainly localized on the nearest-neighbor carbon atoms is supported both by the size of the ${ }^{14} \mathrm{~N}$ quadrupole interaction and by the ${ }^{13} \mathrm{C}$ hyperfine interaction. We detect hyperfine satellites from two different sets of $\mathrm{C}$ neighbors: one due to three equivalent atoms and the other due to six equivalent atoms. The experimentally determined hyperfine parameters are in good agreement with recent results from DFT calculations. ${ }^{5}$ However, we have not observed the predicted hyperfine interaction from a second set of three carbon neighbors.

The position and intensity of the electron-nuclear double spin-flip transitions together with the observation that the sign of the quadrupole interaction is negative for defects contain substitutional nitrogen, conclusively determine the sign of the zero-field splitting to be positive. We furthermore show that the zero-field splitting must be due to anisotropic exchange. An anisotropy of the order of $0.01 \%$ of the experimentally determined ground to first-excited-state splitting ${ }^{1}$ is sufficient to account for the ground-state zero-field splitting.

With the results presented in this paper there is now good accord between experiment and theory for the ground state of the $\mathrm{NV}^{-}$center. However, there is still much to be learned about the excited states. ${ }^{13}$

\section{ACKNOWLEDGMENT}

The authors thank Joh Hansen of E6 Ltd. for the synthesis of the sample.

*m.e.newton@warwick.ac.uk

${ }^{1}$ J. H. N. Loubser and J. A. van Wyk, in Diamond Research 1977, edited by P. Daniel (De Beers Industrial Diamond Division, Ascot, 1977), pp. 11-14.

${ }^{2}$ F. T. Charnock and T. A. Kennedy, Phys. Rev. B 64, 041201(R) (2001).

${ }^{3}$ A. Beveratos, S. Kühn, R. Brouri, T. Gacoin, J.-P. Poizat, and P. Grangier, Eur. Phys. J. D 18, 191 (2002).

${ }^{4}$ S. Felton, A. M. Edmonds, M. E. Newton, P. M. Martineau, D. Fisher, and D. J. Twitchen, Phys. Rev. B 77, 081201(R) (2008).

${ }^{5}$ A. Gali, M. Fyta, and E. Kaxiras, Phys. Rev. B 77, 155206 (2008).

${ }^{6}$ C. A. Coulson and M. J. Kearsley, Proc. R. Soc. London, Ser. A 241, 433 (1957).

${ }^{7}$ G. Davies and M. F. Hamer, Proc. R. Soc. London, Ser. A 348, 285 (1976)

${ }^{8}$ D. A. Redman, S. Brown, R. H. Sands, and S. C. Rand, Phys. Rev. Lett. 67, 3420 (1991).

${ }^{9}$ N. R. S. Reddy, N. B. Manson, and E. R. Krausz, J. Lumin. 38, 46 (1987).

${ }^{10}$ J. P. Goss, R. Jones, S. J. Breuer, P. R. Briddon, and S. Öberg, Phys. Rev. Lett. 77, 3041 (1996).

${ }^{11}$ N. B. Manson and R. L. McMurtrie, J. Lumin. 127, 98 (2007).

${ }^{12}$ J. Harrison, M. J. Sellars, and N. B. Manson, J. Lumin. 107, 245 (2004).

${ }^{13}$ L. J. Rogers, S. Armstrong, M. J. Sellars, and N. B. Manson, arXiv:0806.0895v1 (unpublished).

${ }^{14}$ F. Jelezko, T. Gaebel, I. Popa, A. Gruber, and J. Wrachtrup, Phys. Rev. Lett. 92, 076401 (2004).

${ }^{15}$ G. Davies, S. C. Lawson, A. T. Collins, A. Mainwood, and S. J. Sharp, Phys. Rev. B 46, 13157 (1992).

${ }^{16}$ T. A. Kennedy, J. S. Colton, J. E. Butler, R. C. Linares, and P. J.

Doering, Appl. Phys. Lett. 83, 4190 (2003).

${ }^{17}$ S. C. Lawson, D. Fisher, D. C. Hunt, and M. E. Newton, J. Phys.: Condens. Matter 10, 6171 (1998).

${ }^{18}$ J. Meijer, B. Burchard, M. Domhan, C. Wittmann, T. Gaebel, I. Popa, F. Jelezko, and J. Wrachtrup, Appl. Phys. Lett. 87, 261909 (2005).

${ }^{19}$ A. T. Collins, H. Kanda, and H. Kitawaki, Diamond Relat. Mater. 9, 113 (2000).

${ }^{20}$ L. S. Hounsome, R. Jones, P. M. Martineau, D. Fisher, M. J. Shaw, P. R. Briddon, and S. Öberg, Phys. Status Solidi C 4, 2950 (2007).

${ }^{21}$ D. C. Hunt, D. J. Twitchen, M. E. Newton, J. M. Baker, T. R. Anthony, W. F. Banholzer, and S. S. Vagarali, Phys. Rev. B 61, 3863 (2000).

${ }^{22}$ C. V. H. Stroemann, F. Tshisikhawe, J. O. Hansen, and R. C. Burns, International Patent Application No. WO2006061672 (pending).

${ }^{23}$ O. D. Tucker, Ph.D. thesis, University of Oxford, 1995.

${ }^{24}$ X. F. He, N. B. Manson, and P. T. H. Fisk, Phys. Rev. B 47, 8816 (1993).

${ }^{25}$ J. Harrison, M. J. Sellars, and N. B. Manson, Diamond Relat. Mater. 15, 586 (2006).

${ }^{26}$ O. D. Tucker, M. E. Newton, and J. M. Baker, Phys. Rev. B 50, 15586 (1994).

${ }^{27}$ M. Gerloch, Orbitals, Terms and States (Wiley, Chichester, 1986).

${ }^{28}$ D. R. Lide, CRC Handbook of Chemistry and Physics, 87th ed. (CRC, Boca Raton, FL, 2006).

${ }^{29}$ J. Loubser and J. A. van Wyk, Rep. Prog. Phys. 41, 1201 (1978).

${ }^{30}$ J. R. Morton and K. F. Preston, J. Magn. Reson. (1969-1992) 30, 577 (1978). 